\title{
Third-order energy derivative corrections to the Kohn-Sham orbital hardness tensor
}

\author{
TZONKA MINEVA \\ Institute of Catalysis, Bulgarian Academy of Science (BAS), G. Bonchev Str. 11, 1113-Sofia, Bulgaria \\ e-mail: tmineva@ic.bas.bg
}

\begin{abstract}
The third term in the Taylor expansion of the total energy functional around the number of electrons $N$ is evaluated as the second-order derivative of orbital Kohn-Sham energies with respect to orbital occupancy. Present approach is an extension of an efficient algorithm to compute densityfunctional based orbital reactivity indices. Various energy derivatives used to approximate orbital reactivity indices are defined within the space spanned by the orbital occupation numbers and the KohnSham one-electron energies. The third-order energy functional derivative has to be considered for singular hardness tensor $([\eta])$. On the contrary, this term has negligible influence on the reactivity index values for atomic or molecular systems with positively defined hardness tensors. In this context, stability of a system in equilibrium state estimated through the eigenvalues of $[\eta]$ is discussed. Numerical illustration of the Kohn-Sham energy functional derivatives in orbital resolution up to the third order is shown for benchmark molecules such as $\mathrm{H}_{2} \mathrm{O}, \mathrm{H}_{2} \mathrm{~S}$, and $\mathrm{OH}^{-}$.
\end{abstract}

Keywords. Orbital hardness tensor; DFT; third-order energy derivatives; Janak's theorem.

\section{Introduction}

The conceptual power of density functional theory ${ }^{1}$ (DFT) is nowadays well recognized along with its computational success to describe accurately structural and spectroscopic properties of molecules and solid state systems. Indeed, this is the theory that has made it possible to define rigorously intuitive concepts as hardness or softness, originally introduced by Pearson ${ }^{2}$ in order to provide insight into the nature of chemical reactivity. Starting from the pioneering work of Parr and co-workers ${ }^{3}$, where the chemical potential $(\mu)$ and the hardness $(\eta)$ were first defined within the DFT as first- and second-order KohnSham (KS) total energy derivatives to the number of electrons $N$, many researchers have further developed methods to assess the chemical reactivity indices. So it became possible to assign numbers to the reactivity indices computed from first principles and to exploit these values to rationalize wide range of chemical interactions, ranging from the atomic and molecular reactions, ${ }^{4}$ surface adsorption processes. ${ }^{5}$ and nanoscale objects. ${ }^{6}$ Exhaustive descriptions of the reactivity index applications in physics and chemistry can be found in a monograph of Pearson ${ }^{7}$ and in recent review articles. ${ }^{8,9}$

As the chemical potential is a global quantity for a chemical system, much more attention has been given to the hardness that characterizes the local response at a given point inside the molecular region. Among various working formulae $e^{10-27}$ that were proposed to approximate total and local hardness the definition of hardness tensor [ç] was introduced ${ }^{27-33}$ as well. Computational schemes using charge sensitivity analysis ${ }^{29,30}$ whereas the diagonal hardness tensor elements $\left(c_{i j}\right)$ were obtained from the semiempirical schemes ${ }^{31,32}$ were initially proposed. Atoms in molecule (AIM) methods ${ }^{32}$ were also adopted. Later, several works ${ }^{15,16,33-36}$ appeared which promoted the idea to define $\eta_{i j}$ in its "natural" framework, namely within DFT. Neshev and Proynov, ${ }^{33,34}$ adopted the $X_{\alpha}$ approximation to derive $\eta_{i j}$ as first derivative of the orbital energy with respect to the orbital occupation. Further, this idea was generalized using Janak's theorem ${ }^{37}$ for the density functional computational methods. ${ }^{15,16,35,36}$

The interest to hardness tensor computations is mainly due to the fact that it provides the basis to obtain other reactivity indices as softness tensor, total softness and hardness, and the Fukui functions. In self-consistent charge schemes, popular for example in semi-empirical and tight-binding methods, the diagonal elements of the atomic hardness tensor give the change of Coulomb energy of an orbital with respect to its occupation number. ${ }^{38}$ Orbitally resolved hardness tensor algorithm ${ }^{16,39}$ (ORHT) was incorpo- 
rated into a DFT-TB hybrid method implemented ${ }^{40}$ into the deMon ${ }^{41}$ code, which uses atomic hardness tensor elements $c_{i i .}{ }^{42}$

Nevertheless the concept of hardness tensor was largely used less attention was given to hardness tensor variation with the change of $N$. A stability of a molecular system studied through the hardness tensor eignevalues is also less discussed. It is known ${ }^{43}$ that for zero or negative eigenvalues of $[\eta]$ is necessary to account also for the third term in the Taylor expansion of the total energy functional around the number of electrons. Therefore, in this paper we will focus on the computations of the tensor $[\mathbf{A}]$, which elements defines the $\eta_{i j}$ variations with respect to the occupation numbers $n_{k}$. An extension of the ORHT scheme that includes the third-order KS energy derivative to the orbital occupancy into the computations of other reactivity indices, such as orbital Fukui indices, orbital, local and total softness, and total hardness, is proposed as well.

\section{Kohn-Sham one-electron energy derivatives within the ORHT scheme}

Density $(\rho(\mathbf{r}))$ change caused by external perturbations is often studied in the framework of DFT through expansion of the total energy functional around the number of electrons $N$ in a Taylor series. Following Janak's extension of DFT for fractional occupations, the energy functional can be expanded around the state, characterized by the equilibrium values of occupation numbers $n^{0}\left(n_{1}^{0}, n_{2}^{0}, \ldots, n_{k}^{0}\right)$ and by the corresponding KS-eigenvalues $\varepsilon^{0}=\left(\varepsilon_{1}^{0} \ldots \varepsilon_{k}^{0}\right)$.

$$
\begin{aligned}
\Delta E & =\left(\frac{\partial E}{\partial N}\right) \Delta N+\frac{1}{2}\left(\frac{\partial^{2} E}{\partial N^{2}}\right)(\Delta N)^{2}+\ldots \\
= & \sum_{i} \frac{\partial E}{\partial n_{i}} \Delta n_{i}+\frac{1}{2} \sum_{i j} \frac{\partial^{2} E}{\partial n_{i} \partial n_{j}} \Delta n_{i} \Delta n_{j} \\
& +\frac{1}{6} \sum_{i j k} \frac{\partial^{3} E}{\partial n_{i} \partial n_{j} \partial n_{k}} \Delta n_{i} \Delta n_{j} \Delta n_{k}+\ldots
\end{aligned}
$$

where $\Delta n_{i}=n_{i}-n_{i}^{0}$. In Janak's formulation, ${ }^{37}$ first derivatives of the total energy with respect to the occupation numbers $n_{i}$ give the Kohn-Sham oneparticle orbital energies. Second term in the Taylor expansion, (1), defines hardness tensor: ${ }^{27}$

$$
\partial^{2} E / \partial n_{i} \partial n_{j}=\eta_{i j}
$$

According to the above formula the hardness tensor elements are positive and the hardness tensor is a symmetric tensor. Applying Janak's theorem the $i j$ th element of $[\eta]$ can be obtained as the first derivative of $\varepsilon_{i}$ with respect to $n_{j}{ }^{15,16}$

$$
\partial \varepsilon_{i} / \partial n_{j}=\eta_{i j} .
$$

It is worth emphasizing that the use of Janak's extension of DFT in our approach has two advantages: (1) the DF-energy functional can be expanded over the non-integer occupation numbers; and (2) in the calculation of hardness matrix elements one takes only first-order derivatives, thus diminishing the numerical errors. Therefore, the condition of having a symmetric hardness tensor is fulfilled with fair accuracy (mostly up to the third digit) depending, of course, on the particular molecular system. Hardness tensor elements are themselves approximations to the hardness kernel, ${ }^{44}$

$$
\eta\left(\mathbf{r}, \mathbf{r}^{\prime}\right)=\frac{\delta^{2} F[\rho]}{\delta \rho(\mathbf{r}) \delta \rho\left(\mathbf{r}^{\prime}\right)},
$$

where $F[\rho]$ is the Hohenberg-Kohn universal functional. ${ }^{1}$

The third order derivative in (1)

$$
\frac{\partial^{3} E}{\partial n_{i} \partial n_{j} \partial n_{k}}=\frac{\partial^{2} \varepsilon_{i}}{\partial n_{i} \partial n_{j}}=A_{i j}
$$

measures the variation of $\eta_{i j}$ upon small changes in orbital occupation numbers. Usually, third term in (1) is neglected as being much smaller than the second one. If, the Taylor series is truncated at the second term (linear approximation), the energy behavior around the equilibrium state, $\left(\varepsilon_{i}^{0}, n_{i}^{0}\right)$, can be studied topologically not as a function of the position in the real molecular space but as a function of the eigenvalues and occupation numbers. The search for the extreme of the energy functional $\Delta E(n, \varepsilon)$ upon the density variation leads to a linear system of equations as follows:

$$
\frac{\partial \Delta E}{\partial n_{i}}=\varepsilon_{i}+\sum_{j}^{N} \eta_{i j} \Delta n_{j}=0, \quad i=1 \ldots N .
$$

The solution of (6) at given $\varepsilon_{i}$ and with $\operatorname{Det}\left(\eta_{i j}\right) \neq 0$ with respect to $\Delta n_{i}$ gives: 


$$
\Delta n_{i}=-\sum_{j=1}^{N}[\eta]_{i j}^{-1} \varepsilon_{j}=-\sum_{j=1}^{N} s_{i j} \varepsilon_{j}
$$

with $s_{i j}$ being the softness tensor elements, that are identical with the elements of the inverse hardness tensor.

Considering a system with a fixed deviation of the $i$-th occupation number from its equilibrium value, $\sum_{i} \Delta n_{i}=\Delta N$, the set of (6) becomes:

$$
\begin{aligned}
\varepsilon_{i}+\sum_{j=1}^{N} \eta_{i j} \Delta n_{j}+\lambda & =0, \quad i=1, \ldots, N, \\
\sum_{i} \Delta n_{i} & =\Delta N .
\end{aligned}
$$

In the last equation, $\lambda$ is the Lagrange multiplier and can be interpreted as the effective electronegativity, or the negative of the chemical potential. Since the Kohn-Sham orbital energies can be understand as orbital electronegativity ${ }^{15,16,39}$ by taking the derivative of $\lambda$ relative to $\varepsilon_{i},(8)$, one approximates the orbital Fukui indices.

$$
f_{i}=\frac{\partial \lambda}{\partial \varepsilon_{i}}=\frac{\partial n_{i}}{\partial N}, \quad \sum_{i} f_{i}=1
$$

The relation between the orbital Fukui indices and the orbital softness, $s_{i}=\sum_{j} s_{i j}$, is:

$$
f_{i}=\left(\frac{\partial n_{i}}{\partial \mu}\right)\left(\frac{\partial \mu}{\partial N}\right)=\eta s_{i} .
$$

As the softness is an additive quantity the total hardness is defined as:

$$
\eta=\frac{1}{S}=1 / \sum_{i j} s_{i j}=1 / \sum\left[\eta_{i j}\right]^{-1}
$$

Equations (6)-(11) provide an operational scheme to compute orbital reactivity indices as orbital responses, in the vicinity of the system equilibrium point toward small external density perturbations in the space spanned by the orbital occupation numbers and the Kohn-Sham one-electron energies.

It is worth to note that the above equations are applicable for cases, whereas the hardness tensor is non-singular. According to the Mors lemma, ${ }^{43}$ only for $\operatorname{Det}\left(\eta_{i j}\right) \neq 0$ the higher order terms in the Taylor series do not change the properties of the energy functional, $\Delta E(n, \varepsilon)$.
If the third term in (1) is considered under the assumption of $\Delta n_{j}=\Delta n_{k}(6)$ turns into:

$$
\frac{\partial \Delta E}{\partial n_{i}}=\varepsilon_{i}+\sum_{j=1}^{N} \Delta n_{j}\left(\eta_{i j}+A_{i j} \Delta n_{j}\right)=0, \quad i=1 \ldots N .
$$

It is possible to introduce now a corrected hardness tensor $\left[\eta^{\mathrm{C}}\right]$ with elements

$$
c_{i j}^{C}=\left(c_{i j}+A_{i j} \Delta n_{j}\right),
$$

and to apply straightforwardly the (6)-(11) to compute reactive indices taking into account the third order KS-energy functional derivative.

Numerically, orbital hardness tensor elements $\left(\eta_{i j}\right)$ and their variation with respect to $\Delta n_{j}\left(A_{i j}\right)$ are obtained with sufficient accuracy from the finitedifference formulae:

$$
\begin{aligned}
\eta_{i j} & =\frac{\partial^{2} E}{\partial n_{i} \partial n_{j}}=\frac{\partial \varepsilon_{i}}{\partial n_{j}} \\
& =\lim _{\Delta n_{j} \rightarrow 0} \frac{\varepsilon_{i}\left(n_{j}-\Delta n_{j}\right)-\varepsilon_{i}\left(n_{j}\right)}{\Delta n_{j}}, \\
A_{i j} & =\frac{\partial^{2} \varepsilon_{i}}{\partial^{2} n_{j}} \\
& =\lim _{\Delta n_{j} \rightarrow 0} \frac{\varepsilon_{i}\left(n_{j}-\Delta n_{j}\right)-2 \varepsilon_{i}\left(n_{j}-1 / 2 \Delta n_{j}\right)+\varepsilon_{i}\left(n_{j}\right)}{1 / 2\left(\Delta n_{j}\right)^{2}} .
\end{aligned}
$$

To carry out numerically the difference quotient of (14) and (15) with finite $n_{j}$ one first provides selfconsistent calculations for the ground-state energy and then for each perturbed orbital with occupation $n_{j}=1-n_{j}$ and $n_{j}=1-1 / 2 \quad n_{j}$ for the occupied orbitals and $n_{j}=0+n_{j}$ and $n_{j}=0+1 / 2 n_{j}$, for the virtual orbitals.

\section{Positively defined hardness tensor and stability of a molecular system}

As already mentioned, the third term in the Taylor series, (1), appears to be important for singular [ $\eta]$. Therefore, we find it of interest to discuss below the stability of a many-electron system in terms of eigenvalues of the hardness tensor.

System stability is determined in general by the eigenvalues of the matrix whose elements are energy 
derivatives of second order (Hessian) with respect to the coordinates or to the occupation numbers, ${ }^{45}$ i.e. in our case the eigenvalues of hardness tensor. The eigenvalues $\zeta_{i}$ and the eigenvectors $c_{i j}$ are obtained by taking orthogonal transformation of $[\eta]$,

$$
[\eta]^{\mathrm{N}}=[\mathbf{C}]^{\prime}[\eta][\mathbf{C}],
$$

with $[\mathbf{C}]$ being an orthogonal matrix and $[\eta]^{\mathrm{N}}-$ the normal presentation of the hardness tensor. It is straightforward to define now the normal coordinates in the parametric space of occupation numbers and KS-one-particle energies as: $Q_{i}=\sum_{j} c_{i j} \Delta n_{j}$ and to express $\eta_{i j}$ in terms of $\zeta_{i}$ and $c_{i j}: \eta_{i j}=\sum_{k} \zeta_{k} c_{k i} c_{k j}$. Positively defined hardness tensor $\left(\zeta_{i}>0\right.$, for each $i=1, \ldots, N)$ means a minimum of energy variation $\Delta E(n, \varepsilon)$, and thus stable molecular systems. For, $\zeta_{k} \quad 0$ the system would lose its stability and $A_{i j}$ corrections to the hardness tensor would need to be taken into account.

In the limit of total hardness approximation $\eta=1 / 2(I-A)$ where only two (HOMO and LUMO) orbitals are considered with $\varepsilon_{\mathrm{HOMO}}=-I$ and $\varepsilon_{\mathrm{LUMO}}=$ $-A$, orbital hardness elements are defined from the Pariser ${ }^{46}$ formula as $\eta_{\mathrm{II}}=\eta_{\mathrm{AA}}=I-A$ and the offdiagonal hardness tensor elements, $\eta_{\mathrm{IA}}$, are set equal to zero, the eigenvalues of this two-dimensional hardness tensor is equal to $2 \eta$. Therefore, for this particular case of taking into account only the frontier orbitals, the higher hardness value is directly associated with the higher system stability. This is not necessarily true if the whole spectrum of the molecular system is considered in reactivity index computations. Moreover, $\eta$ is a size dependent quantity and cannot be used in general as the only stability criterion.

\section{The third-order corrections to $\eta$ and $f_{i}$ for $\mathrm{H}_{2} \mathrm{O}, \mathrm{H}_{2} \mathrm{~S}$ and $\mathrm{OH}^{-}$}

Present calculations were performed using the deMon code and the GGA functional as proposed by Perdew ${ }^{47}$ for correlation and by Perdew and Wang ${ }^{48}$ for exchange energies and potentials (PW86). Orbital basis functions of double- $\zeta$ (DZVP) quality ${ }^{49}$ were adopted, whereas the auxiliary functions for fitting the density include orbitals up to $l=4$. Computations of first- and second-order one-particle energy derivatives were carried out assuming $\Delta n_{i}=0.5$ and 0.25 respectively.
Recently reported ${ }^{42,50}$ benchmark computations with deMon code on hardness tensors and reactivity indices revealed very good numerical stability towards basis sets and exchange-correlation functionals. The symmetry of the hardness tensor is maintained, even though its elements are computed differently, either using the left side (occupied) or right side (unoccupied) derivative of the one-particle energies with respect to the orbital occupation numbers.

In tables $1 \mathrm{a}$ and $1 \mathrm{~b}$ are reported $\eta_{i j}$ together with $A_{i j}$ values of the occupied valence orbitals and LUMO for $\mathrm{H}_{2} \mathrm{O}$ and $\mathrm{H}_{2} \mathrm{~S}$ molecules. Table 1c collects $\eta_{i j}$ and $A_{i j}$ for the occupied valence $\mathrm{OH}^{-}$orbitals. Note that the maximum asymmetry for $\eta_{i j}$ is found equal to $10^{-3}$, which is a very reasonable accuracy for these quantities. For $[\mathbf{A}]$ asymmetry of about $10^{-1}$ is obtained and the $A_{i j}$ elements in table 1 are the symmetriesed values. Our computations for the second and third terms in the Taylor series, (1), reveal that $A_{i j}$ values amount to a maximum of $22 \%$ of the orbital hardnesses for the occupied orbitals of water molecule and to $36 \%$ of the LUMO diagonal hardness element (see table $1 \mathrm{a}$ ). The $A_{i j} / \eta_{i j}$ ratio is slightly smaller for $\mathrm{H}_{2} \mathrm{~S}$ molecule, while for $\mathrm{OH}^{-} A_{i j}$ are found to be equal or less than $10 \%$ of the orbital hardnesses.

As expected, the minimum eigenvalue of the hardness tensor $\zeta_{\min }$ is positive, that is $\zeta_{\min }=0.32,0.63$ and $0.45 \mathrm{eV}$ for $\mathrm{H}_{2} \mathrm{O}, \mathrm{H}_{2} \mathrm{~S}$ and $\mathrm{OH}^{-}$, respectively. To examine the influence of $A_{i j}$ in computing Fukui indices, (10), and total hardness (11) we performed the calculations using both $\eta_{i j}$ and $\eta_{i j}{ }^{C}$. As seen from the $f_{i}$ and $\eta$ values reported in table 2 the third term in the Taylor expansion of the KS-energy functional is insignificant. Indeed, the relative trend of the orbital Fukui index values $\left(f_{i}^{*}\right)$ coming from computations with the third-order corrections to [ $\eta$ ] follows those of the linear fukui indices $\left(f_{i}\right)$, though there is a small difference between the absolute values of $f_{i}$ and $f_{i}^{*}$, especially in the case of $\mathrm{H}_{2} \mathrm{O}$ molecule. For water molecule the diagonal hardness element of the LUMO undergoes higher variation (higher $A_{4 A_{1}, 4 A_{1}}$ value) when compared to the $A_{4 A_{1}, 4 A_{1}}$ value for $\mathrm{H}_{2} \mathrm{~S}$. In spite of the small numerical difference found between $f_{i}$ and $f_{i}^{*}$ the chemical behavior of the orbitals remains unaffected by the third-order correction.

For the three species the third-order corrections did not stabilize/destabilize additional the hardness tensors, as very similar results for $\zeta_{i}$ were obtained from both types of computations: with and without accounting for $A_{i j}$. Our numerical results corroborate 
Table 1(a). Hardness tensor elements $\eta_{i j}$ and their variations $A_{i j}$ with the occupation numbers for the valence occupied orbitals and the LUMO of $\mathrm{H}_{2} \mathrm{O}$ molecule. The results are in $\mathrm{eV}$.

\begin{tabular}{|c|c|c|c|c|c|c|c|c|c|c|}
\hline & \multicolumn{5}{|c|}{$\eta_{i j}$} & \multicolumn{5}{|c|}{$A_{i j}$} \\
\hline & $2 \mathrm{~A}_{1}$ & $1 \mathrm{~B}_{2}$ & $3 \mathrm{~A}_{1}$ & $1 \mathrm{~B}_{1}$ & $4 \mathrm{~A}_{1}$ & $2 \mathrm{~A}_{1}$ & $1 \mathrm{~B}_{2}$ & $3 \mathrm{~A}_{1}$ & $1 \mathrm{~B}_{1}$ & $4 \mathrm{~A}_{1}$ \\
\hline $2 \mathrm{~A}_{1}$ & $10 \cdot 06$ & $9 \cdot 34$ & $8 \cdot 50$ & 8.49 & $5 \cdot 64$ & $-2 \cdot 16$ & $-1 \cdot 60$ & $-2 \cdot 05$ & $-2 \cdot 10$ & -0.55 \\
\hline $1 B_{2}$ & & $9 \cdot 30$ & 8.03 & $8 \cdot 13$ & 5.46 & & -1.47 & -1.94 & $-2 \cdot 08$ & -0.42 \\
\hline $3 \mathrm{~A}_{1}$ & & & 9.29 & $8 \cdot 15$ & $4 \cdot 83$ & & & -1.93 & $-2 \cdot 20$ & -0.23 \\
\hline $1 \mathrm{~B}_{1}$ & & & & $9 \cdot 10$ & $5 \cdot 00$ & & & & $-2 \cdot 31$ & -0.38 \\
\hline $4 \mathrm{~A}_{1}$ & & & & & $6 \cdot 37$ & & & & & $-2 \cdot 32$ \\
\hline
\end{tabular}

Table 1(b). Hardness tensor elements $\eta_{i j}$ and their variations $A_{i j}$ with the occupation numbers for the valence occupied orbitals and the LUMO of $\mathrm{H}_{2} \mathrm{~S}$ molecule. The results are in $\mathrm{eV}$.

\begin{tabular}{|c|c|c|c|c|c|c|c|c|c|c|}
\hline & \multicolumn{5}{|c|}{$\eta_{i j}$} & \multicolumn{5}{|c|}{$A_{i j}$} \\
\hline & $2 \mathrm{~A}_{1}$ & $1 \mathrm{~B}_{2}$ & $3 \mathrm{~A}_{1}$ & $1 \mathrm{~B}_{1}$ & $4 \mathrm{~A}_{1}$ & $2 \mathrm{~A}_{1}$ & $1 B_{2}$ & $3 \mathrm{~A}_{1}$ & $1 \mathrm{~B}_{1}$ & $4 \mathrm{~A}_{1}$ \\
\hline $2 \mathrm{~A}_{1}$ & $8 \cdot 31$ & $6 \cdot 36$ & $5 \cdot 87$ & $5 \cdot 84$ & $5 \cdot 04$ & -1.05 & -0.89 & -0.81 & -1.05 & -0.61 \\
\hline $1 \mathrm{~B}_{2}$ & & $6 \cdot 47$ & $5 \cdot 40$ & $5 \cdot 21$ & 4.99 & & -0.82 & -0.82 & -0.90 & -0.54 \\
\hline $3 \mathrm{~A}_{1}$ & & & $6 \cdot 05$ & $5 \cdot 32$ & $4 \cdot 87$ & & & -0.82 & -0.94 & -0.54 \\
\hline $1 \mathrm{~B}_{1}$ & & & & 5.95 & $4 \cdot 38$ & & & & -0.92 & -0.48 \\
\hline $4 \mathrm{~A}_{1}$ & & & & & $7 \cdot 23$ & & & & & $-2 \cdot 42$ \\
\hline
\end{tabular}

Table 1(c). Hardness tensor elements $\eta_{i j}$ and their variations $A_{i j}$ with the occupation numbers for the valence occupied orbitals of $\mathrm{OH}^{-}$. The results are in $\mathrm{eV}$.

\begin{tabular}{cccccccc}
\hline & \multicolumn{3}{c}{$\eta_{i j}$} & & \multicolumn{3}{c}{$A_{i j}$} \\
\cline { 2 - 3 } \cline { 6 - 8 } & $2 \sigma$ & $3 \sigma$ & $1 \pi$ & & $2 \sigma$ & $3 \sigma$ & $1 \pi$ \\
\hline $2 \sigma$ & 13.64 & $12 \cdot 11$ & 12.37 & & 1.29 & 1.13 & 1.16 \\
$3 \sigma$ & & 12.41 & 11.49 & & 1.17 & 1.08 \\
$1 \pi$ & & & 12.07 & & & 1.02 \\
\hline
\end{tabular}

Table 2. Orbital Fukui indices $\left(f_{i}\right)$ for valence occupied orbitals and total hardness $(\eta)$ in $\mathrm{eV}$ computed without $A_{i j}$ corrections for $\mathrm{H}_{2} \mathrm{O}, \mathrm{H}_{2} \mathrm{~S}$ and $\mathrm{OH}^{-} . f_{i}^{*}$ and $\eta^{*}$ indicates orbital fukui indices and total hardness in $\mathrm{eV}$ for $\mathrm{H}_{2} \mathrm{O}$, $\mathrm{H}_{2} \mathrm{~S}$ and $\mathrm{OH}^{-}$computed by employing the $A_{i j}$ corrections to the hardness tensor.

\begin{tabular}{|c|c|c|c|c|c|}
\hline Orbital & $2 \mathrm{~A}_{1}$ & $1 \mathrm{~B}_{2}$ & $3 \mathrm{~A}_{1}$ & $1 \mathrm{~B}_{1}$ & $4 \mathrm{~A}_{1}$ \\
\hline \multicolumn{6}{|l|}{$\mathrm{H}_{2} \mathrm{O}$} \\
\hline$f_{i}$ & -0.496 & $0 \cdot 255$ & $0 \cdot 297$ & $0 \cdot 192$ & 0.753 \\
\hline$f_{i}^{*}$ & $-0 \cdot 384$ & $0 \cdot 191$ & $0 \cdot 216$ & $0 \cdot 158$ & 0.919 \\
\hline$\eta / \eta *$ & $5 \cdot 78 / 5 \cdot 44$ & & & & \\
\hline \multicolumn{6}{|l|}{$\mathrm{H}_{2} \mathrm{~S}$} \\
\hline$f_{i}$ & -0.205 & $0 \cdot 237$ & $0 \cdot 199$ & $0 \cdot 501$ & $0 \cdot 268$ \\
\hline$f_{i}^{*}$ & -0.199 & $0 \cdot 183$ & $0 \cdot 116$ & $0 \cdot 505$ & $0 \cdot 396$ \\
\hline$\eta / \eta *$ & $5.25 / 5.22$ & & & & \\
\hline Orbital & $2 \sigma$ & & $3 \sigma$ & & $1 \pi$ \\
\hline \multicolumn{6}{|l|}{${ }^{\mathrm{a}} \mathrm{OH}^{-}$} \\
\hline$f_{i}$ & \multicolumn{2}{|c|}{$-0 \cdot 474$} & 0.492 & \multicolumn{2}{|c|}{$0 \cdot 982$} \\
\hline$f_{i}^{*}$ & \multicolumn{2}{|c|}{-0.473} & $0 \cdot 491$ & \multicolumn{2}{|c|}{0.982} \\
\hline$\eta / \eta^{*}$ & \multicolumn{2}{|c|}{$11 \cdot 64 / 11 \cdot 92$} & & & \\
\hline
\end{tabular}

the conclusion that the third order KS energy functional derivative does not affect the reactivity indices for systems characterized by a positively defined hardness tensor.

\section{Conclusions}

An extension of the numerical algorithm to compute orbital reactivity indices (ORHT) including the third-order KS energy functional derivative is proposed. This scheme is easily achieved numerically as it uses the Janak's theorem within DFT, thus reducing the order of the derivatives. The third term in the Taylor expansion of KS energy over the number of electrons can be neglected for positively defined hardness tensors, as demonstrated numerically for $\mathrm{H}_{2} \mathrm{O}, \mathrm{H}_{2} \mathrm{~S}$ and $\mathrm{OH}^{-}$. This term, however, might affect significantly the results for less stable systems, for which zero or even negative eigenvalues of [ $\eta]$ appear. Same conclusion applies for reactivity index 
methods based on the use of hardness tensor within the electronegativity equalization method in spite of the numerical approach to the hardness tensor elements.

\section{Acknowledgement}

The author is grateful to the Bulgarian National Science Council for financial support vide grant no. HT 2-01/2003.

\section{References}

1. Hohenberg P and Kohn W 1964 Phys. Rev. B136 864; Kohn W and Sham L 1965 Phys. Rev. A140 113

2. Pearson R G 1963 J. Am. Chem. Soc. 853533

3. Parr R G, Donnelly R A, Levy M and Palke W E J 1978 Chem. Phys. 683801

4. Chattaraj P K and Maiti B J 2001 Phys. Chem. A105 169

5. Wilke S, Cohen M H and Scheffler M 1996 Phys. Rev. Lett. 771560

6. Sabin J R, Trickey S B, Apell S P and Oddershede J 2000 Int. J. Quantum Chem. 77358

7. Pearson R G 1997 Chemical hardness: Applications from molecules to solids (Weinheim: Wiley-VCH)

8. Chermette H 1999 J. Comput. Chem. 20129

9. Geerlings P, De Proft F and Langenaeker W 2003 Chem. Rev. 1031793

10. Yang W and Mortier W J 1986 J. Am. Chem. Soc. 108 5708

11. Cohen M H 1986 In Topics in current chemistry, Vol 183: Density functional theory IV - Theory of chemical reactivity (ed.) R F Nalewajski (Heidelberg: SpringerVerlag) p. 143

12. Gazquez J L, Vela A and Galwan M 1987 In Structure and bonding, Vol. 66: Electronegativity (ed.) K Sen (Heidelberg: Springer-Verlag) p. 79

13. Komorowski L and Lipinski 1991 J. Chem. Phys. 157 45; Balawender R and Komorwski L 1998 J. Chem. Phys. 1095203

14. Cioslowski J and Mixon S T $1991 \mathrm{~J}$. Am. Chem. Soc. 1134142

15. Neshev N and Mineva T 1996 In Metal-ligand interactions: Structures and reactivity (eds) N Russo and D R Salahub (Dordrecht: Kluwer) p. 361

16. Neshev N, Mineva T and Parvanov V 1996 In Heterogeneous catalysis, Proc. 8th Int. Symp. Heterogeneous Catalysis, Varna, p. 67-72; ibidem p. 79-84

17. Senet P 1996 J. Chem. Phys. 105 6471; Senet P 1997 J. Chem. Phys. 1072516

18. Liu G H 1997 J. Chem. Phys. 106165

19. Pearson R G 1997 Chemical hardness: Applications from molecules to solids (Weinheim: Wiley-VCH) and references therein

20. Grigorov M, Weber J, Chermette $\mathrm{H}$ and Tronchet $\mathrm{J}$ M J 1997 Int. J. Quantum Chem. 61551
21. De Proft F, Liu S, Geerlings P, Parr R G and Pol S 1998 J. Chem. 721737

22. Gilardoni F, Weber J, Chermette H and Ward T R 1998 J. Phys. Chem. A102 3607

23. Roy R K, Krishnamurti S, Geerlings P and Pal S 1998 J. Phys. Chem. A102 3746

24. Nalewajski R F 1993 Struct. Bonding 80115

25. Fuentealba P, Perez P and Contreras R 2000 J. Chem. Phys. 1132544

26. Meness L, Tiznado W, Contreras $\mathrm{R}$ and Fuentealba $\mathrm{P}$ 2004 Chem. Phys. Lett. 383181

27. Liu G H and Parr R G 1995 J. Am. Chem. Soc. 117 3179

28. Nalewajski R F 1984 J. Am. Chem. Soc. 944106

29. Nalewajski R F 1985 J. Phys. Chem. 892831

30. Nalewajski R F 1993 In Structure and bonding: vol. 80 Chemical hardness (ed.) K Sen (Heidelberg: SpringerVerlag) p. 115

31. Baekelandt, Mortier W J and Schoonheydt R A 1993 In Structure and bonding: vol. 80 Chemical hardness (ed.) K Sen (Heidelberg: Springer-Verlag) p. 187

32. Komorowski L 1997 Int. J. Quantum Chem. 61499

33. Neshev N M and Proynov E I 1987 In Heterogeneous catalysis. Proc. 8th Int. Symp. Heterogeneous Catalysis, Sofia, part I, p. 342

34. Neshev N M and Proynov E I 1989 J. Mol. Catal. 1989 54484

35. Grigorov M, Weber J, Chermette $\mathrm{H}$ and Tronchet $\mathbf{J}$ M J 1997 Int. J. Quantum Chem. 61551

36. Grigorov M, Weber J, Vulliermet N, Chermette H and Tronchet J M J 1998 J. Chem. Phys. 1088790

37. Janak J F 1978 Phys. Rev. B18 7165

38. Elstner M, Porezag D, Jungnickel G, Elsner J, Haugk, M, Frauenheim T, Suhai S and Seifert G 1998 Phys. Rev. B58 7260

39. Mineva T, Neshev N, Russo N, Sicilia E and Toscano M 1999 Adv. Quantum Chem. 33273

40. Duarte H A, Heine T and Seifert G 2005 Theor. Chem. Acc. 11468

41. Köster A M, Geudtner G, Goursot A, Heine T, Patchkovskii S, Vela A and Salahub D 2004 deMon Program, Version 1.0.4gkd

42. Mineva T and Heine T 2005 Int. J. Quant. Chem. (in press)

43. Gilmore R 1981 Catastrophe theory for scientists and engineers (New York: J. Wiley and Sons) ch. 10

44. Parr R G and Yang W 1989 Density functional theory of atoms and molecules (New York: Oxford University Press)

45. Mineva T, Parvanov V, Petrov I, Neshev N and Russo N 2001 J. Phys. Chem. A105 1959

46. Pariser R 1953 J. Chem. Phys. 21568

47. Perdew J P 1986 Phys. Rev. B33 8822

48. Perdew J P and Wang Y 1986 Phys. Rev. B33 8800

49. Godbout N, Salahub D R, Andzelm J and Wimmer E 1992 Can. J. Phys. 70560

50. Mineva T and Heine T 2004 J. Phys. Chem. A108 11086 